\title{
APONTAMENTOS EM FILOSOFIA DA LÓGICA
}

\author{
Notes on Philosophy of Logic
}

Gilson Maicá de Oliveira ${ }^{1}$

\begin{abstract}
RESUMO: Neste artigo discutimos alguns aspectos da lógica nos dias atuais. O propósito central é mostrar a evolução dessa disciplina. Começamos com uma breve introdução onde especificamos o que queremos dizer com o termo "lógica". A seguir, exporemos e discutiremos o que consideramos ser algumas das principais áreas de investigação da lógica atual. Concluímos o artigo com algumas observações sobre lógicas não clássicas e seus impactos sobre a filosofia. Ao final do texto se encontram mais detalhes que apontam para um aprofundamento e uma ampliação das questões aqui tratadas.
\end{abstract}

PALAVRAS-CHAVE: Lógica; Lógica matemática; Áreas da lógica; Lógicas não clássicas; Filosofia da lógica.

\begin{abstract}
In this paper we discuss some aspects of logic in present days. The primary proposal is to show the evolution of this discipline. We begin with a brief introduction where we sketch what we mean by the term "logic". In the next section we explore and discuss what we consider some of the key research areas of current logic. Finally, we conclude with some observations about non-classical logics and its impacts on philosophy. For a detail research about logic and others aspects related to it, see the references at the end of the paper.
\end{abstract}

KEY-WORDS: Logic; Mathematical logic; Areas of logic; Non-classical logic; Philosophy of logic.

A lógica é uma disciplina que evoluiu enormemente nos últimos dois séculos e, ao que tudo indica, continuará a sofrer modificações significativas. Três aspectos devem ser observados na análise do estado atual da lógica, e que contribuíram para este estado de coisas: (1) primeiro, o amálgama sofrido entre lógica e matemática a partir de meados século XIX, particularmente pelas contribuições de matemáticos como G. Boole e G. Frege, que propiciaram um aumento significativo de seu escopo teórico; (2) segundo, sua

\footnotetext{
${ }^{1}$ Doutor em Filosofia pela Universidade Federal de Santa Catarina (UFSC), leciona lógica e filosofia da ciência na Faculdade São Basílio Magno (FASBAM). E-mail: filogmaica@gmail.com
} 
relativização com o surgimento das chamadas lógicas não clássicas, especialmente aqueles sistemas de lógica que de alguma forma rivalizam com o sistema clássico, isto é, as lógicas ditas heterodoxas e, por fim; (3) suas aplicações práticas, tanto na matemática com fora dela, com especial destaque na computação e Inteligência Artificial, sem porém deixar de lado outras áreas como a ciência cognitiva, a linguística, a física e até mesmo a biologia e o direito. Embora, para alguns possa soar estranho, até mesmo a filosofia se beneficiou com o advento da lógica simbólica, ganhando especialmente no rigor de suas investigações e elaborações teóricas. Vamos no que segue discutir brevemente o que queremos dizer quando usamos o vocábulo 'lógica', haja vista seu caráter ambíguo e por vezes nebuloso.

De início devemos deixar claro que a palavra 'lógica' é frequentemente usada em diversas acepções. Interessa-nos destacar aqui duas: ${ }^{2}$ a lógica como disciplina teórica, e a lógica como sinônimo de sistema lógico, designando neste caso um sistema de lógica particular $l$, ou seja, um sistema de cânones de inferência. Aqui comparece uma das noções centrais da lógica, isto é, a noção de consequência lógica. É neste último sentido, como evidenciaremos adiante, que intervém provavelmente uma das mais significativas revoluções na história desta disciplina, o surgimento de sistemas não clássicos.

Usualmente quando nos envolvemos com a caracterização de qualquer disciplina teórica, temos de dar conta de pelo menos dois aspectos: (a) o escopo, objeto ou domínio de investigação da disciplina em foco; (b) o método através do qual a disciplina investiga seu domínio ou objeto. Vamos no que segue discorrer brevemente sobre o escopo da lógica, porém, sem levar a cabo qualquer discussão sobre seu método, o método axiomático, que deixaremos para exposição em outra ocasião. De qualquer forma, o leitor interessado poderá consultar ${ }^{3}$.

Uma definição precisa do escopo da lógica (como disciplina) está completamente fora de cogitação pelo menos por duas razões: (1) suas fronteiras com disciplinas correlatas não são suficientemente nítidas para que se possa delimitar de forma precisa seu campo de atuação. Por exemplo, existem sistemas formais que são por vezes considerados pertencentes ao escopo da lógica e, por vezes, pertencentes à certas áreas da matemática. De fato, embora a matemática e a lógica sejam disciplinas distintas, suas fronteiras, em

\footnotetext{
${ }^{2}$ Naturalmente, o termo "lógica" está relacionado ao que aqui discutimos, mas a lógica também é muito mais do que isso, embora não pretendamos esgotar num artigo introdutório, de caráter didático, todas as suas facetas.

${ }^{3}$ CASSINI, A. El Juego de los Principios: una introducción al método axiomático. Buenos Aires: AZEditora, 2008.
} 
certa acepção, são atualmente difíceis de mapear. Aliás, a questão de quais sistemas formais são sistemas lógicos é uma questão que extrapola o âmbito próprio da lógica, constituindo-se num problema de filosofia da lógica, que não tencionamos aqui discutir com requinte de detalhe. (2) A lógica, como qualquer outro ramo da ciência está em constante transformação, isto é, não se constitui por um conhecimento acabado, como advogou, por exemplo, Kant, ${ }^{4}$ mas que capitaliza com frequência novas áreas de investigação. Aliás, podemos dizer que este é um aspecto que torna a lógica uma disciplina viva e fecunda.

Assim não é possível apresentar uma definição rigorosa e abrangente do que seja a lógica atualmente ${ }^{5}$, haja vista que esta disciplina se constitui numa ciência dinâmica, em constante transformação, e com fronteiras não muito nítidas. Por outro lado, sem muito rigor, podemos dizer afim de qualificar nosso discurso, que a lógica é uma disciplina teórica cujo objetivo é o estudo de certas estruturas abstratas e, sob certo aspecto, não se distingue significativamente de outras disciplinas matemáticas, como a álgebra, a topologia ou a análise. A lógica trata, entre outras coisas, de certas estruturas, os sistemas lógicos, assim como a álgebra trata de outras, como as estruturas de grupo, espaço vetorial e corpo. Alguns textos apresentam a lógica como a ciência que investiga as "formas válidas de inferência". A despeito de tal estudo tenha motivado o aparecimento da lógica como disciplina teórica, não reflete mais o que se tem feito em lógica em nossos dias. ${ }^{6}$

Os desenvolvimentos que se fizeram em lógica a partir do século XIX e, especialmente no século XX, provocaram mudanças radicais e profundas nesta ciência. Em parte, podese dizer que isto está associado ao fato de a lógica hoje possuir uma grande variedade de aplicações, tanto teóricas, como na matemática e em outras ciências; quanto práticas, como na ciência da computação e na inteligência artificial. Assim sendo, a lógica passou a constituir disciplina indispensável a qualquer um que pretenda se dedicar seriamente

\footnotetext{
${ }^{4}$ No prefácio da segunda edição de sua Crítica da Razão Pura, Kant afirma que a lógica é uma disciplina que desde Aristóteles não pode dar nenhum passo para trás ou para frente, simplesmente por se constituir numa ciência completa e acabada. (cf. KANT, I. Crítica da Razão Pura. São Paulo: Nova Cultura, 1996, p. 35).

${ }_{5}^{5}$ Para o lógico Elliot Mendelson, a melhor forma de entender uma disciplina, como a lógica, é trabalhar como ela, estudá-la. (cf. MENDELSON, Elliot. Introduction to Mathematical Logic. New York: Van Nostrand Reinhold Company, 1964, p.1). Naturalmente, isto requer certo esforço e dedicação por parte do noviço, para uns mais para outros menos.

${ }^{6}$ Quine citando Whitehead em $O$ Sentido da Nova Lógica faz a seguinte observação: “A lógica antiga está para a nova lógica, menos como outra ciência anterior, do que como um fragmento pré-científico da mesma disciplina. Nas palavras do próprio Whitehead: 'No desenvolvimento moderno da lógica, a lógica aristotélica tradicional apresenta-se como uma simplificação do problema completo que o assunto comporta. Há nisso, uma analogia com a aritmética das tribos primitivas comparada à matemática moderna"”. (cf. QUINE, W.O. O Sentido da Nova Lógica. 2. ed. Curitiba: Ed. UFPR, 1996, p. 16)
} 
aos fundamentos da ciência ou à sua filosofia. De um modo geral, podemos afirmar que a lógica possui um duplo aspecto:

a) A lógica "pura", que entendemos como o estudo de certas estruturas abstratas, como as estudadas quando se desenvolve sistemas particulares de lógica, como as lógicas intuicionistas, as lógicas polivalentes, as lógicas paraconsistentes ou as lógicas fuzzy. ${ }^{7}$ Ou seja, a lógica "pura" trata do estudo dos sistemas lógicos, das conexões entre eles, de suas vantagens e limitações, sem que se leve em conta, por exemplo, suas possíveis aplicações a outras áreas.

b) A lógica "aplicada", que por seu turno, pode ser dividida em duas esferas: (i) a lógica aplicada às formas válidas de inferência, que por diversas razões, podem ser distintas em áreas distintas de investigação, ou seja, o que é permitido inferir em certos campos do conhecimento, pode não ser em outros. Daí a escolha de um sistema lógico (ou um sistema de cânones de inferência) depender de fatores pragmáticos relativamente ao domínio que se está investigando; (ii) a lógica aplicada as diversas ciências, no sentido em que ela é útil no desenvolvimento de outros campos do saber, por exemplo, a linguística, a computação ou aos fundamentos da física.

A lógica como disciplina teórica envolve atualmente diversas áreas de investigação, entre as quais a teoria da recursão, teoria de modelos, fundamentos da teoria dos conjuntos, teoria das definições, lógica algébrica, teoria de tipos e teoria da prova. ${ }^{8}$ Em cada uma dessas áreas encontram-se problemas lógicos de grande envergadura, como o problema $\mathbf{P} \& \mathbf{N P}$ em teoria da recursão, ou a chamada hipótese do continuum em teoria dos conjuntos.

De um ponto de vista abstrato uma lógica l, (no sentido de sistema lógico), consiste numa estrutura $l=\left\langle F, \vdash_{F}\right\rangle$, onde $F$ é um conjunto não vazio, dito domínio da lógica, cujos elementos são denominados de fórmulas e $\vdash_{F}$ é uma relação sobre $\wp(F) \times F$, dita relação de dedutibilidade ou relação de consequência lógica. Interessa particularmente a caracterização da noção de dedutibilidade, isto é, o que se quer dizer quando se afirma que uma fórmula é dedutível de um conjunto de fórmulas $\Gamma=\left\{\alpha_{1}, \ldots \alpha_{n}\right\}$. O que geralmente escrevemos,

\footnotetext{
${ }^{7}$ Nosso objetivo não é apresentar nenhum desses sistemas, mas tão somente situar o leitor a respeito do estado presente da lógica.

8 O leitor interessado poderá consultar na página do American Mathematical Society o link http://www.ams.org/mathscinet/msc/msc2010.html para ter uma ideia mais precisa das áreas da lógica atual.
} 
ou mais simplesmente,

$$
\Gamma \vdash \alpha .
$$

Entre as propriedades do operador $\vdash$ destacam-se as seguintes:
i. Se $\alpha \in \Gamma$, então $\Gamma \vdash \alpha$. (autodedutibilidade)
ii. $\quad \operatorname{Se} \Gamma \subseteq \Delta \mathrm{e} \Gamma \vdash \alpha$, então $\Delta \vdash \alpha$. (monotonicidade)
iii. $\quad$ Se $\Delta \vdash \alpha$ e de $\Delta \vdash \beta$ para cada $\beta \in \Delta$, então $\Gamma \vdash \alpha$. (regra do corte)
iv. $\quad \Gamma \vdash \alpha$ se existe $\Delta \subseteq \Gamma$ tal que $\Delta \vdash \alpha$. (compacidade)

A forma como se especifica a noção de dedutibilidade é o que distingue um sistema lógico de outro. Com efeito, fala-se atualmente de uma infinidade de lógicas distintas possíveis. ${ }^{9}$ Por exemplo, se $l$ for alguma versão da lógica clássica, podemos então admitir como válidas, do ponto de vista dedutivo, diversas formas de redução ao absurdo, por outro lado, se $l$ é uma lógica intuicionista, como a de Brouwer-Heyting, este tipo de dedução não é aceito. Em lógicas paraconsistentes o princípio de contradição é abandonado, adimitindo-se teoremas contraditórios sem que isso conduza a uma trivialização, um fato absolutamente surpreendente, que não foi percebido antes do surgimento deste tipo de lógica. ${ }^{10}$ A grande variedade de sistemas lógicos existentes naturalmente interessa tanto ao matemático quanto ao filósofo, e em certo sentido, mesmo ao cientista aplicado. O que vale apena neste ponto destacar é o aspecto plural dos sistemas dedutivos, o que evidenciamos com a seguinte observação de Restall: “[...] tenho argumentado que não há uma relação única de consequência lógica, mas sim, há diferentes relações de consequência, que formalizam de modo adequado a noção de validade dedutiva. Não há uma lógica verdadeira, mas sim, muitas." 11

\footnotetext{
${ }^{9}$ Conforme da Costa, quando especificamos quais são as formas válidas de inferência de uma lógica $l$, também estabelecemos aquelas inferências que não são $l$-válidas que chamamos $l$-paralogismos. Na lógica clássica, que aparentemente é a forma mais próxima de como pensamos racionalmente os fenômenos que nos cercam, distinguimos duas formas de paralogismos: as falácias e as induções. As falácias são formas de inferência que reconhecemos como errôneas relativamente aos princípios da lógica clássica, as induções, porém, ainda que não sejam dedutivamente válidas, apresentam certo grau de plausibilidade.

${ }^{10}$ Em nossa dissertação Racionalidade Científica, Paraconsistência e Quase-Verdade, defendemos a tese de que o advento deste tipo de lógicas amplia a noção de racionalidade, haja vista que tradicionalmente muitos filósofos, como Popper, defendiam que o princípio de não-contradição da lógica clássica constituía uma cláusula pétrea da noção de racionalidade. (cf. OLIVEIRA, G. M. Racionalidade Cientifica, Paraconsistência e Quase-Verdade. Florianópolis: Dissertação de Mestrado, 2008.)

${ }^{11}$ Cf. RESTALL, G. Logical Pluralism and the Preservation of Warrants. In: Rahman et al. (eds.), Logic, Epistemology, and the Unity of Science, p. 163.
} 
Queremos igualmente patentear, que existem diversas maneiras de caracterizar um sistema de lógica, e isso depende tanto de fatores epistemológicos quanto pragmáticos. Podemos, por exemplo, ter em mente uma abordagem linguística da lógica, ou uma abordagem algébrico-topológica ou ainda via teoria de categorias. Essas diversas formas de tratar os variados sistemas lógicos, revelam em parte a riqueza e a complexidade dessa disciplina presentemente, bem como sua ampla e rica diversidade teórica. Este estado de coisas é em grande medida fruto da aproximação entre lógica e matemática.

\section{Algumas áreas da lógica}

\subsection{A teoria de modelos}

A teoria de modelos é o estudo das relações entre linguagem e o "mundo", ou mais precisamente entre linguagens formais e interpretações de linguagens formais. A teoria de modelos em seu estágio atual de desenvolvimento é devida aos trabalhos seminais de A. Tarski e A. Robinson na década de 1950. Dentre as contribuições mais significativas da teoria de modelos está a matematização, feita por Tarski, do conceito de verdade. Os trabalhos de Tarski permitem falar da noção de verdade de forma rigorosa, isto é, matemática.

Outro tema de interesse da teoria de modelos e a relação entre consistência, completude e correção de teorias formais. Usualmente tem-se a ideia de que um aparato dedutivo para uma dada linguagem formal (interpretada) deve cumprir a seguinte condição fundamental: se uma sentença é deduzida de um determinado conjunto de sentenças, então esta sentença é verdadeira em todos os modelos dessa teoria. Diz-se, neste caso, que o aparato dedutivo é correto para a semântica em foco. De fato, esta é uma forma de dizer que as deduções preservam a verdade. ${ }^{12}$ Uma questão básica aqui é a seguinte: teorias consistentes sempre possuem modelo? Embora isto seja desejável, não ocorre em todos os casos. Quando ocorre de uma teoria consistente possuir modelo, dizemos que ela é completa, por exemplo, o cálculo de predicados de primeira ordem é completo, o mesmo ocorre com a cálculo proposicional, a lógica intuicionista e diversos sistemas de lógica modal. Porém, este fato não se verifica para a lógica de segunda ordem e em geral para lógicas de ordem superior. Dito de outra forma, nas teorias formais usuais

\footnotetext{
${ }^{12}$ Cf. BRANQUINHO, J. et. al. Enciclopédia de termos lógico-filosóficos. São Paulo: Martins Fontes, 2006 p. 528 .
} 
(fortes) e consistentes, os conceitos de sentença verdadeira e sentença demonstrável (teorema) jamais coincidem, o primeiro sendo mais abrangente que o segundo.

Na teoria de modelos também se estudam as propriedades de Löwenheim-Skolem e da compacidade. A primeira propriedade diz que se uma teoria possui modelo, então tem um modelo cujo domínio é finito ou enumerável. Por compacidade deve-se entender que se todo subconjunto finito de sentenças de dada teoria formal tem um modelo, então a teoria tem modelo. É importante notar que estas propriedades fazem parte do escopo do cálculo de predicados.

A teoria de modelos possui muitas aplicações, tanto na matemática pura quanto na filosofia. Particularmente se destacam suas aplicações na filosofia da ciência e na teoria do conhecimento.

\subsection{A teoria da recursão}

Em poucas palavras, a teoria da recursão trata do que é exequível mecanicamente, computacionalmente, isto é, sem recurso à inteligência humana. Em certa acepção é uma teoria geral das máquinas, que atuam de forma mecânica, sempre na dependência de comandos. Esta teoria estuda certas máquinas ideais introduzidas por Alan Turing. Entre as questões típicas dessa teoria está a das funções recursivas. Todos os computadores atualmente construídos são realizações físicas das máquinas de Turing. Quando os primeiros computadores começaram a ser construídos, por volta de 1950, com John Von Neumann e seu grupo, a teoria geral das máquinas já existia, pois Turing, e independentemente Post, já haviam estabelecido suas bases em 1936. Alan Turing, por volta de 1935, quando ainda era estudante do King's College, em Cambridge, propôs solucionar o chamado problema da decisão (Entscheidurngsproblem) proposto originalmente por Hilbert. Este problema consiste em indagar se existe um procedimento efetivo (mecânico) para determinar se todos os enunciados matemáticos verdadeiros podem ou não ser provados.

Num sentido preciso, uma máquina de Turing é um modelo abstrato de um computador, que se restringe apenas aos aspectos lógicos do seu funcionamento (memória, estados e transições) e não à sua implementação física. Numa máquina de Turing pode-se modelar qualquer computador digital.

Na teoria da recursão, um dos resultados mais importantes é o teorema de ChurchTuring, segundo o qual, para a aritmética tradicional, suposta consistente, não existe uma 
máquina de Turing capaz de provar todos os seus teoremas e somente eles. Como corolário conclui-se que a atividade humana é ainda indispensável para o desenvolvimento do conhecimento matemático em particular e supostamente para o conhecimento científico em geral.

\subsection{Teoria das definições}

A teoria das definições ao lado da teoria da prova constitui uma das operações fundamentais da lógica. Historicamente existem tantos tipos de definições, que não existe até o presente momento nenhuma teoria abrangente que envolva de forma harmônica todas as definições. Por exemplo, em filosofia fala-se de definições nominais e definições reais, as primeiras relacionadas a termos de uma teoria, a segunda, relacionada a objetos reais (físicos). Na matemática encontram-se definições por composição de funções, por meio da recorrência, tanto finita como transfinita, por postulados (ou implícitas), indutivas e ampliadoras da linguagem etc. Em ciências como a física encontram-se definições operacionais e ostensivas etc. Em síntese, não parece haver uma teoria abrangente das definições usadas na ciência.

Grosso modo, uma definição é a especificação da natureza de algo. Chamamos definiendum ao que se quer definir e definiens ao que se define. Por exemplo, pode-se definir solteiro como homem não casado. Escrevemos:

\section{definiendum $\stackrel{\text { def }}{=}$ definiens}

para exprimir a relação entre definiendum e definiens.

Se restringirmos nosso estudo da teoria das definições às ciências formais (lógica e matemática), então temos duas classes de definições: as abreviativas e as ampliativas. As primeiras constituem processos que visão simplesmente auxiliar na exposição de teorias formais, não ampliando suas linguagens. Neste caso temos duas categorias de definições: as simples, que substituem grupos complexos de símbolos por um símbolo novo, e as contextuais, que introduzem símbolos novos, como abreviações, em certos contextos. Em princípios essas definições são elimináveis. As definições ampliativas, estudadas originalmente por Łukasiewicz, ampliam a linguagem na qual comparecem. 
Como se vê a teoria das definições tem uma importância estratégica tanto nas ciências formais quanto nas ciências empíricas e está relacionada fortemente, no caso da lógica e da matemática, ao método axiomático.

\subsection{Lógica algébrica}

Nesta área da lógica usam-se métodos algébricos para tratar de sistemas lógicos. $\mathrm{O}$ tratamento algébrico da lógica tem em Leibniz um dos precursores, contudo, foi com De Moragan e Boole, em meados do século XIX, que uma perspectiva algébrica da lógica ganhou fôlego. Podemos afiançar, de qualquer forma, que o grande desenvolvimento da lógica algébrica veio a ocorrer com os trabalhos de Alfred Tarski e Paul Halmos. Vale lembrar que esta forma de tratar os sistemas lógicos é a abordagem preferida da chamada escola polonesa de lógica, de grande influência nesta área. Um exemplo típico desta perspectiva em lógica é a abordagem algébrica do cálculo proposicional clássico, dada pelo que chamamos álgebra de boole. Em certo sentido, trabalhar com o cálculo proposicional nada mais é do que trabalhar com uma álgebra de boole.

De um modo geral, as estruturas algébricas que comparecem na lógica clássica resultam do processo de passagem ao quociente: tem-se um sistema lógico, escolhe-se uma relação de equivalência conveniente, isto é, compatível com as noções lógicas básicas e passa-se ao quociente, obtendo-se desse modo a estrutura que algebriza o sistema. Desse modo, por exemplo, mostra-se que a álgebra de Boole e a álgebra de Heiting ${ }^{13}$ constituem algebrizações, do cálculo proposicional clássico e do cálculo proposicional intuicionista respectivamente.

O método proposto acima para a algebrização do cálculo proposicional clássico e intuicionista, porém, não se presta a todos os casos, por exemplo, muitos sistemas de lógicas não clássicas, como o cálculo paraconsistente, não pode ser algebrizado por esse método, haja vista que neste sistema lógico não há nenhuma relação de congruência significativa. Além disso, mesmo no quadro clássico, a passagem ao quociente não permite tratar 'algebricamente' determinados conceitos lógicos, como os tableaux de Smullyan. Nestes casos, a utilização de pré-álgebras torna-se significativo: primeiro quando não há uma relação de congruência, ou quando a relação de equivalência estabelecida não é compatível com todas as operações lógicas; segundo, em casos em

13 Uma exposição elementar desses sistemas encontra-se em MIRAGLIA, Francisco. Cálculo Proposicional: uma interpretação da álgebra e da lógica. Campinas: Unicamp, 1987. 
que, mesmo dispondo de uma congruência, não se deseja passar o quociente para não ocultar fatos significativos desse sistema lógico. No primeiro caso temos estruturas que denominamos álgebras de Curry, ${ }^{14}$ introduzidas por Newton da Costa afim de sistematizar uma teoria geral da algebrização dos sistemas lógicos; no segundo, lançamos mão de préálgebras no sentido usual deste termo. ${ }^{15}$

\section{As lógicas não-clássicas}

I can't belive THAT! Said Alice.

Can't you? The Queen said a pitying tone. Try again: draw a long breath, and shut your eyes. Alice laughed. There's no use trying, she said one CAN'T belive impossible things.

I daresay you haven't hat much practice, said the Queen. When I was your age, I always did it for half-an-hour a day. Why, sometimes I've belived as many as six impossible things before breakfast.

Lewis Carroll

Não constitui nenhum exagero afirmar que o surgimento das lógicas não-clássicas representa uma das revoluções conceituais mais significativas de nosso tempo. Uma apresentação, classificação e avaliação do status desses sistemas de lógica, implicaria necessariamente um esforço hercúleo, que demandaria um trabalho bem mais extenso, dada a amplitude, multiplicidade e profundidade de tais lógicas. Assim sendo, nos propomos aqui um esquema sumário, com alguma discussão epistemológica em torno de sua relevância filosófica.

O raciocínio dedutivo não se enquadra, completamente, na lógica elementar clássica. $\mathrm{Na}$ matemática, por exemplo, tem-se necessidade de recursos lógicos mais potentes. Sistemas de lógica não elementar esbarram com um obstáculo extremamente difícil de ser superado: os paradoxos lógicos. No âmbito clássico é possível reforçar a lógica elementar por duas vias distintas: pela teoria de conjuntos axiomatizada, ou pelo cálculo de predicados de ordem superior (teoria de tipos). Efetivamente esses sistemas de lógica não resolvem, stricto sensu, o problema dos paradoxos; tão-somente em tais sistemas aparentemente não ressurgem, em virtude de se recorrer a expedientes ad hoc para contorná-los. Além disso, deve-se ter em mente que as limitações da lógica elementar não advêm tão somente do fato de seu aparato dedutivo não dar conta da matemática usual,

\footnotetext{
${ }^{14}$ Certas estruturas receberam o nome do lógico norte-americano H.B. Curry, porque foi ele um dos primeiros a defender o uso sistemátcio de pré-álgebras no domínio da lógica.

${ }^{15}$ Cf. KRAUSE, Décio. Introdução aos Fundamentos Axiomáticos da Ciência. São Paulo: EPU, 2002, p. 36.
} 
mas também que se pode construir sistemas dedutivos divergentes entre si, o que nos leva a admitir que não há um conceito único e definitivo de consequência lógica. A possibilidade de elaborar sistemas lógicos mais fortes, do ponto de vista sintático ou dedutivo, ou de sistemas que divergem do clássico em sua semântica ou estrutura dedutiva, foi o ponto de partida para a construção do que denominamos lógicas não clássicas, que em certo sentido, podem ou completar a lógica clássica, lógicas não clássicas ortodoxas, ou rivalizar com ela, as chamadas lógicas heterodoxas.

Existem atualmente muitos sistemas lógicos e, desde o surgimento do aparato lógico 'clássico' tem-se defendido a necessidade de melhorá-lo, modificá-lo, ou até mesmo substituí-lo por sistemas mais potentes ou que deem conta de aspectos do processo dedutivo que aquele ignora. Um exemplo típico disso pode ser tirado dos debates em torno do condicional material. Proposto de início pelos estóicos, a 'implicação material' foi formalizada por Frege (1879) e Russell \& Whitehead (1910), e provida de uma semântica por Post (1921) e Wittgenstein (1922). Porém, já McColl em 1880 defendia a necessidade de um condicional mais estrito; a 'implicação estrita' formalizada por Lewis (1918). Posteriormente, novos debates em torno da implicação estrita, deram origem a 'implicação relevante' e a lógica relevante. ${ }^{16}$

Apresentar uma distinção clara entre lógicas clássicas e lógicas não clássicas não constituir tarefa fácil, pode-se delinear, de qualquer forma, alguns critérios de ordem didática que nos forneçam alguma visibilidade sobre o assunto. De fato, é possível afirmar que as lógicas não clássicas divergem das lógicas clássicas, ${ }^{17} \mathrm{em}$ maior ou menor grau, em um ou mais dos seguintes aspectos: sintático, dedutivo ou semântico. Vamos apresentar uma distinção entre lógicas não clássicas e lógicas clássicas, tendo em mente estes aspectos.

Assim sendo, diremos que um sistema lógico $l$ é não-clássico relativamente a um sistema clássico, se e só se cumpre um ou mais dos seguintes requisitos:

I. l possui uma linguagem mais rica em capacidade de expressão, permitindo que se trate de conceitos que não são formalizáveis no escopo de uma sistema lógico clássico. Podemos arrolar como exemplos neste caso os sistemas de lógica modal

\footnotetext{
${ }^{16}$ Cf. HAACK, S. Filosofia das Lógicas. São Paulo: Unesp, 2002, p. 207.

${ }^{17}$ Devemos ter em conta que não há uma lógica clássica, mas muitos sistemas clássicos, como o cálculo proposicional clássico, o cálculo de predicados (como ou sem igualdade), diversos sistemas de ordem superior (teoria de tipos) e até mesmo diversos sistemas de teoria de conjuntos, que em certo sentido também são sistemas lógicos. Efetivamente, mesmo uma caracterização do que seria um sistema 'clássico' constitui um empreendimento difícil de equacionar com precisão.
} 
alética, em que se introduzem os conceitos de necessidade $\square$ e possibilidade $\diamond$; e o sistema de lógica deôntica, em que comparecem os operadores obrigatório $O$ e permitido $P$. Outros exemplos são a lógica temporal ou cronológica, cultivada por A. N. Prior e a lógica epistêmica desenvolvida principalmente por Lewis (1912) e continuada por S. Kripke.

II. $l$ possui uma semântica distinta dos sistemas lógicos clássicos, isto é, $l$ é um sistema fundado em teorias de conjuntos distintas das usuais. Nas lógicas para-completas, por exemplo, não é válido o princípio de terceiro excluído. Particularmente em lógicas polivalentes uma proposição pode assumir outros valores de verdade entre 1 (verdadeiro) e o 0 (falso).

III. $l$ diverge dos sistemas clássicos por derrogar um ou mais princípios clássicos. Dentre estes princípios clássicos temos, por exemplo, o princípio de identidade, de não-contradição e terceiro excluído. Vale lembrar, que embora estes princípios sejam os mais citados na literatura, não são os únicos que alicerçam os sistemas clássicos. As lógicas que derrogam tais princípios são usualmente chamadas de lógicas heterodoxas, entre elas encontram-se as lógicas não-reflexivas, em particular as lógicas de Schrödinger que derrogam o princípio de identidade; as lógicas paraconsistentes que abandonam o princípio de não-contradição e as lógicas para-completas, como as intuicionistas e as lógicas polivalentes, em que não vale o princípio de terceiro excluído.

Como já dissemos, não é possível estabelecer com rigor absoluto uma distinção entre lógicas não clássicas e lógicas clássicas. Por exemplo, alguns sistemas de lógica nãoreflexiva e paraconsistentes podem, de certo ponto de vista, ser consideradas extensões da lógica clássica. Podemos também ter sistemas modais paraconsistentes e lógicas deônticas paraconsistentes. Também podemos pensar em lógicas epistêmicas polivalentes, lógicas epistêmicas cronológicas etc.

Vamos no que segue apresentar, como estudo de caso, três tipos de lógicas, usualmente admitidas como não clássicas, que consideramos interessantes para ilustrar o estado atual destes sistemas de lógica. 


\subsection{Estudo de caso: As lógicas paracompletas intuicionista de Brower e Heyting e polivalente de Lukasiewicz}

As lógicas para-completas são sistemas lógicos que derrogam o princípio de terceiro excluído. Nestes sistemas de lógica ou em teorias fundamentadas em tais lógicas, podemos ter proposições que nem elas nem suas negações sejam verdadeiras. Dois exemplos de lógicas paracompletas são as lógicas polivalentes de Łukasiewicz e a lógica intuicionista de L.E. J. Brower e A. Heyting.

Jean Łukasiewicz (1922) construiu sistemas de lógicas polivalentes afim de investigar proposições modais e as noções de possibilidade e necessidade intimamente conectadas a tais proposições. Efetivamente, questões conectadas já haviam sido aventadas por Aristóteles em seu Da Interpretação, onde discute o problema da verdade de proposições sobre o futuro, por exemplo, a célebre proposição “Amanhã haverá uma batalha naval”. Esta proposição, de acordo com uma lógica bivalente, ou é verdadeira ou é falsa, não havendo uma terceira possibilidade. De qualquer modo, se ela for verdadeira ou falsa, o futuro estará previamente determinado e, portanto, vivemos num mundo determinístico, caso seja possível estabelecer um terceiro valor, como indeterminado, então o futuro é contingente e, por conseguinte, a referida proposição não é nem verdadeira, nem falsa. Naturalmente esta questão envolve intrincadas questões metafísicas, que não pretendemos discutir aqui. Por outro lado, ela demonstrar que o princípio de terceiro excluído de sistemas clássicos não é auto-evidente.

Assim sendo, Łukasiewicz propõe um sistema de lógica em que dada uma proposição $\alpha$ temos como valores de verdade o verdadeiro $v(\alpha)=1$, o falso $v(\alpha)=0$ e o indeterminado, denotado por $v(\alpha)=1 / 2$. A partir disso, Łukasiewicz introduz seu cálculo proposicional tivalente $\iota_{3}$ através da matriz $M=\langle\{0,1 / 2,1\}, \neg, \rightarrow\rangle$, onde $\{0,1 / 2,1\}$ são os valores de verdade, $\{1\}$ o conjunto de valores distinguidos de verdade e os operadores $\neg \mathrm{e} \rightarrow$ são definidos por meio das seguintes tabelas:

\begin{tabular}{lcrrrr}
$\alpha$ & $\neg \alpha$ & $\alpha \rightarrow \beta$ & 0 & $1 / 2$ & 1 \\
\hline 0 & 1 & 0 & 1 & 1 & 1 \\
$1 / 2$ & $1 / 2$ & $1 / 2$ & $1 / 2$ & 1 & 1 \\
1 & 1 & 1 & 0 & $1 / 2$ & 1
\end{tabular}

O conectivo de possibilidade $P$ é definido do seguinte modo:

$$
P(\alpha) \stackrel{\text { def }}{=} \neg \alpha \rightarrow \alpha
$$


Os demais conectivos lógicos, conjunção, disjunção, e o bicondicional são definidos a partir dos anteriores.

Łukasiewicz posteriormente (1922) generalizou seu cálculo proposicional para uma lógica polivalente com infinitos valores de verdade. Além disso, definiu uma família $t_{n}$ de sistema polivalentes com $n$ valores de verdade, com $2 \leq n \leq \aleph_{\omega}$. Também E. Post, independentemente dos trabalhos de Łukasiewicz, e motivado inicialmente por questões de ordem puramente técnica, propôs em 1921 um sistema de lógica $n$-valente, hoje conhecido como lógica de Post.

É interessante observar que o cálculo proposicional bivalente de Łukasiewicz é equivalente ao cálculo proposicional clássico e, além disso, todos os sistemas de lógica $n$-valentes de Łukasiewicz, com $n$ finito ou infinito, são subsistemas do cálculo proposicional clássico. Também é digno de nota o fato desses sistemas terem recebido um tratamento axiomático, primeiro o cálculo trivalente $\iota_{3}$ e mais tarde $\bigsqcup_{n}$ (com $n$ finito) pelas mãos de Wajsberg (1935). G. Moisil (1940), tendo em vista as relações bastante conhecidas entre o cálculo proposicional clássico e as álgebras de Boole, promoveu o estudo de estruturas algébricas relacionadas aos cálculos proposicionais $n$-valentes de Łukasiewicz, com \$n\$ finito, as quais denominou álgebras de kukasiewicz. Uma exposição e discussão pormenorizada de todas as implicações dos sistemas de lógica de Łukasiewicz naturalmente demandaria muito mais do que aqui apresentamos, de qualquer modo, fica patente a relevância desses sistemas para a matemática e filosofia atuais. Particularmente se destacam suas aplicações à teoria dos circuitos elétricos, à linguística, à programação de computadores e à teoria das probabilidades. Alguns autores chegaram a desenvolver aplicações das lógicas trivalentes à Mecânica Quântica, com profundas implicações sobre a filosofia da ciência e a metafísica, ainda não completamente investigadas pelos filósofos. Para o leitor interessado em se aprofundar no tema indicamos o livro de G. Malinowski. ${ }^{18}$

Outros sistemas de lógica para-completas são os sistemas intuicionistas, que embora sejam aparentadas com os sistemas de Łukasiewicz, por derrogarem o princípio de terceiro excluído, possuem motivação filosófica distinta.

A lógica intuicionista desenvolveu-se originalmente a partir da filosofia da matemática, particularmente de questões relacionadas a legitimidade de demonstrações

${ }^{18}$ Cf. MALINOWSKI, G. Many-Valued Logics. Oxford: Oxford University Press, 1993, bem como Many Valued Logic de N. Rescher (Cf. RESCHER, N. Many Valued Logic. Columbus: McGraw-Hill, 1969). 
não-construtivas em matemática. Para matemáticos como Leopold Kronecker e Henri Poincaré, dentre outros, a matemática não pode ser reduzida à teoria dos conjuntos, tal como proposto por G. Cantor. Kronecker, em particular, defendia que a noção de número natural deveria ser entendida como a categoria mais fundamental da matemática, o que ficou patenteado em sua célebre afirmação de que Deus nos deu os números naturais e todo o resto seria obra do homem. Também Poincaré, na mesma linha, considerava a indução matemática, um dos axiomas da aritmética de Peano, como a intuição básica da matemática, sobre a qual esta disciplina deveria ser construída.

Em 1912, o matemático holandês L. E. J. Brower, em seu trabalho de doutoramento, acabou apresentando os fundamentos do intuicionismo, que desenvolveu nos anos subsequentes. Em síntese, a tese broweriana consiste em negar a matemática clássica e a possibilidade de se realizar provas por redução ao absurdo. Assim, na matemática usual, quando pretendemos provar que existe um objeto $x$ que tem certa propriedade $P$, o que escrevemos $\exists x P(x)$, via de regra partimos da suposição da não existência, ou seja, assumimos a negação $\neg \exists P(x)$. A partir dessa hipótese, em com recursos da teoria na qual estamos trabalhando (seus postulados), derivamos duas proposições contraditórias, digamos $\alpha$ e $\neg \alpha$. Conforme os cânones da lógica clássica, isso nos permite derivar uma contradição na teoria, isto é, algo como $(\neg \alpha \wedge \alpha)$. Dado que supõe-se que nenhuma proposição verdadeira possa implicar uma contradição, descarta-se nossa hipótese como falsa e, consequentemente, a proposição original que pretendíamos provar é admitida como verdadeira.

Para Brower, este tipo de demonstração não pode ser aceita. Para ele, ao provarmos a falsidade da hipótese $\neg \alpha$, acabamos provando que $\neg \neg \alpha$ é verdadeira, mas não que $\alpha$ é verdadeira, ou seja, não é lícito para os intuicionistas o princípio clássico da dupla negação, a sabe: $\neg \neg \alpha \rightarrow \alpha$. A razão da negativa da dupla negação como prova de que $\neg \neg \alpha$ fornece uma prova para $\alpha$ está no fato de termos de admitir que em todos os casos há uma prova de $\alpha$ ou de $\neg \alpha$. A hipótese de que não chegamos a uma prova de $\neg \alpha$, somos obrigados a reconhecer que $\alpha$, recai no princípio de terceiro excluído, ou seja, $(\alpha \vee \neg \alpha)$, o que também não é admitido pelos intuicionistas. Para Brower, a matemática era uma atividade essencialmente mental e o formalismo da lógica e matemática seriam decorrentes dessa atividade. Assim, para ele, o que há em matemática é aquilo que pode ser 'construído' pela inteligência humana a partir de certas intuições fundamentais, como por exemplo, a intuição da unidade, ou seja, temos a intuição do que seja um objeto, e a 
partir disso, reiteradamente, construímos a ideia de dois, três ou quatro objetos e assim por diante. Não dispomos, porém, da intuição de uma totalidade infinita. O infinito, para Brower e os intuicionistas, só existe em potência, já que podemos construir tantos objetos quanto desejarmos, mas nunca uma totalidade infinita deles, como pretendeu G. Cantor.

A lógica intuicionista, em sua formulação primeira, constitui realização do matemático russo A. Kolmogorov (1925), que pretendeu dar conta formalmente das ideias de Brower. Depois de Kolmogorov, tem especial destaque na lógica intuicionista Arend Heyting, que propôs seu sistema em um artigo de 1930. Vamos apresentar aqui a axiomática de Heyting para o cálculo proposicional, afim de ilustrar nossa apresentação.

Heyting utiliza letras latinas minúsculas como variáveis proposicionais, adotando $\neg$, $\wedge, \mathrm{V}, \rightarrow \mathrm{e} \leftrightarrow$ como conectivos primitivos. A única regra de inferência é modus ponens. A lista de postulados adotados é relativamente longa, havendo onze formulas ao todo, que Heyting demonstra ser independentes. Os axiomas são os seguintes:

$$
\begin{aligned}
& \text { H.1. } a \rightarrow(a \wedge a) \\
& \text { H.2. }(a \wedge b) \rightarrow(b \wedge a) \\
& \text { H.3. }(a \rightarrow b) \rightarrow((a \wedge c) \rightarrow(b \wedge c)) \\
& \text { H.4. }((a \rightarrow b) \wedge(b \rightarrow c)) \rightarrow(a \rightarrow c) \\
& \text { H.5. } b \rightarrow(a \rightarrow b) \\
& \text { H.6. }(a \wedge(a \rightarrow b)) \rightarrow b \\
& \text { H.7. } a \rightarrow(a \vee b) \\
& \text { H.8. }(a \vee b) \rightarrow(b \vee a) \\
& \text { H.9. }((a \rightarrow c) \wedge(b \rightarrow c)) \rightarrow((a \vee b) \rightarrow c) \\
& \text { H.10. } \neg a \rightarrow(a \rightarrow b) \\
& \text { H.11. }((a \rightarrow b) \wedge(a \rightarrow \neg b)) \rightarrow \neg a .
\end{aligned}
$$

Muitas das fórmulas do cálculo proposicional clássico são deriváveis no sistema de Heyting; porém, há naturalmente fórmulas que não podem ser obtidas, entre as quais $\neg \neg a \rightarrow a$, que expressa o princípio de dupla negação. Também a fórmula $a \vee \neg a$, que expressa a lei do terceiro excluído, não pode ser derivada no sistema de Heyting.

O sistema de Heyteing não é o único sistema de lógica intuicionista, embora seja o mais consolidado: de fato, a lógica de Johansson (1936), que não tem o décimo axioma, parece segundo S. Haack, consideralmente, ser um melhor pretendente a representar 
apropriadamente os princípios lógicos que são aceitáveis pelos padrões intuicionistas. ${ }^{19}$ De qualquer modo é interessante notar que a lógica de Heyting pode tanto ser encarada como uma extensão da lógica clássica, como uma alternativa, isto é, como uma lógica heterodoxa.

\section{Conclusão}

Algumas das conquistas mais notáveis da filosofia em nosso tempo, dependem de um bom conhecimento da lógica matemática, particularmente de setores de alta complexidade técnica desta disciplina. Lamentavelmente, muitos estudantes e mesmo professores de filosofia têm pouca ou nenhuma bagagem em lógica, o que os tem impossibilitado de compreender em todo seu alcance e profundidade muitas das realizações filosóficas que dependem das ciências formais, particularmente, em epistemologia da ciência, filosofia da matemática, filosofia da mente e teoria do conhecimento de nosso tempo. Nestas caso a atividade filosófica acaba se resumindo a análise de autores do passado, especialmente naqueles temas fortemente relacionadas à epistemologia e a ontologia analítica. De fato, mesmo áreas como a ética e a teoria do direito, tem hoje necessidade das poderosas ferramentas da lógica.

A lógica é hoje um poderoso instrumento de análise do discurso filosófico e científico. É um instrumento de cálculo formal, algoritmo, que procura investigar, entre outras coisas, o que é a demonstrabilidade. Teoria da demonstração, teoria de modelos, teoria da computabilidade, teoria de conjuntos e fundamentos da matemática, são alguns dos ramos de investigação da lógica. Ao filósofo que não tenha familiaridade com as conquistas da lógica matemática, resta, em muitos casos, somente divagar sem bússola no mar da especulação filosófica.

\section{Referências}

BACHELARD, G. La Philosophie du non: Essai d'une philosophie du nouvel esprit scientifique. Paris: Presses Univ. de France, 1966.

BAZHANOV, V.A. Imaginary Geometry of N.I. Łobachevsky and Imaginary Logic of N.A. Vasiliev. In: Modern Logic, v.4, p.148-156, 1994.

${ }^{19}$ Cf. HAACK, S. Filosofia das Lógicas. São Paulo, Unesp, 2002, p. 286. 
BELL, E.T. The Development of Mathematics. McGraw-Hill. 1945.

BENACERRAF, P. \& PUTNAM, H. Philosophy of Mathematics: selected readings. 2nd. ed., New York: Cambridge Un. Press, 1996.

BEZIAU, J-Y. De la logique formelle a la logique abstrate. Boletim da Sociedade Paranaense de Matemática, v. \{\bf 14\}, p.41-50, 1994.

. La Logique abstraute au sein de la mathématique moderne. Ruch Filozofyczne, v.6, 1994.

BLANCHÉ, R. História da Lógica de Aristóteles a Bertrand Russell. Lisboa: Edições 70, 1985.

BOOLE, G. An investigation of the laws of thought, on which are founded the mathematical theories of logic and probabilities. In: Google Books, 1854.

. The Mathematical Analysis of Logic: being an enssay towards a calculus of deductive reasoning. Cambridge: Cambridge Univ. Press, 2009.

BOYER, C.B. The History of Calculus and its Conceptual Development. New York: Dover, 1974.

BRANQUINHO, J. et. All. Enciclopédia de termos lógico-filosóficos. São Paulo: Martins Fontes, 2006.

CANTOR, G. Contributions to the Fouding of the Theory of Transfinite Numbers. New York: Dover, 1955.

CARRION, R. \& da Costa, N.C.A. Introdução à Lógica Elementar. Porto Alegre: Ed. UFGS, 1988.

CASSINI, A. El Juego de los Principios: una introducción al método axiomático. Buenos Aires: AZ-Editora, 2008.

COHEN, P. J. Set Theory and the Continum Hypothesis. New York: Dover, 2008.

CROSSLEY, J. N. [et. al.]. What is Mathematical Logic. New York: Dover, 1990.

COSTA, N.C.A. Ensaio Sobre os Fundamentos da Lógica. São Paulo: Hucitec, 1980. . O Conhecimento Científico. São Paulo: Discurso Editorial, 1997. . Sistema Formais Inconsistentes. Curitiba: Ed. UFPR, 1993.

DAUBEN, J.W. Georg Cantor: his mathematics and philosophy of the infinite. Princeton: Univ. Press, 1990.

FERREIRÓS, J. The Road to Modern Logic - an interpretation. The Bulletin of Symbolic Logic, vol. 7 4, dec. 2001, p.441-487. 
FRAENKEL, A. A. Abstract Set Theory. Netherlands: North-Holland, 1953.

GOLDBLATT, R. Topoi: the categorial analysis of Logic. Mineola: Dover, 2006.

GONSETH, F. La Logique en tant que physique de l'objet quelconque. In Actes du Congrès international de philosophie scientifique, Paris: Hermann, 1936.

HAACK, S. Filosofia das Lógicas. São Paulo: Unesp, 2002.

HALMOS, P. Algebraic Logic. Chelsea Pu. Co., 1962.

HATCHER, W. S. The Logical Foundations of Mathematics. Oxford: Pergamon Press, 1982.

HEIJENOORTH, From Frege to Gödel: a source book in mathematical logic. Cambridge: Harvard Univ. Press, 1967.

HILBERT, D. Mathematical Problems. In: Bulletin of the American Mathematical Society. 37, 2000, p. 407-36.

KLEENE, S.C. Introduction to Metamathematics. Amsterdam: North-Holland, 1952.

KANT, I. Crítica da Razão Pura. São Paulo: Nova Cultura, 1996.

KRAUSE, Décio. Introdução aos Fundamentos Axiomáticos da Ciência. São Paulo: EPU, 2002.

. A Obra de N.C.A. da Costa em Lógica. São Paulo, 1990.

KNEEBONE, G. T. Mathematical Logic and the Foundations of Mathematics: an introductory survey. New York: Dover Publications, 2001.

MAGALHÃES, J. C. M. Axiomatização e Estrutura da Teoria Sintética da Evolução. In: Boletim da Sociedade Paranaense de Matemática, 18, 121-134, 2000.

MALINOWSKI, G. Many-Valued Logics. Oxford: Oxford University Press, 1993.

MCKINSEY, J.C.C., Sugar, A.C. and Suppes, P. Axiomatic Foundations of Classical Particles Mechanics. In: Journal of Rational Mechanics and Analysis, 2, p. 253-273.

MENDELSON, Elliot. Introduction to Mathematical Logic. New York: Van Nostrand Reinhold Company, 1964.

MIRAGLIA, Francisco. Cálculo Proposicional: uma interpretação da álgebra e da lógica. Campinas: Unicamp, 1987.

NAGEL, Ernes \& NEWMAN, James R. Gödel's Proof. New York: New York Univ. Press, 2001. 
OLIVEIRA, G. M. Racionalidade Cientifica, Paraconsistência e Quase-Verdade. Florianópolis: Dissertação de Mestrado, 2008.

. Notas em Lógica e Racionalidade Científica. In: Tabule: Revista de Philosophia, n.5, p. 61-82, 2011.

Mat. 8, 1990.

O Advento da Matemática não-standard. In: Monografias da Soc. Paran.

PALAU, G. Introducción Filosófica a las Lógicas no Clásicas. Barcelona: Gedisa, 2002.

PRIEST, G. An Introduction to Non-Classical Logic. Cambridge: Cambridge Univ. Press, 2001.

RESCHER, N. Many Valued Logic. Columbus: McGraw-Hill, 1969.

REID, C. Hilbert. New York: Springer-Verlag, 1996.

RESTALL, G. Logical Pluralism and the Preservation of Warrants. In. Rahman et al. (eds.), Logic, Epistemology, and the Unity of Science.

QUINE, W.O. O Sentido da Nova Lógica. 2. ed. Curitiba: Ed. UFPR, 1996.

STOLL, R. R. Set Theory and Logic. New York: Dover, 1979.

SUPPES, P. Introduction to Logic. New York: Dover, 1999.

TILES, M. The Philosophy of Set Theory: an historical introduction to Cantor's paradise. New York: Dover, 2004.

WEINGARTNER, P. (Ed.). Alternative Logics: Do science need them. Berlin: SpringerVerlag, 2003. 